OPEN ACCESS

Edited by:

Lina Ma,

Capital Medical University, China

Reviewed by:

Erica D. Diminich,

Stony Brook Medicine, United States

Fei Sun,

Michigan State University,

United States

*Correspondence:

Zhihui Wang

wangzhihui@ncncd.chinacdc.cn

Dantao Peng

pengdantao@163.com

tThese authors have contributed equally to this work

Specialty section:

This article was submitted to Aging and Public Health,

a section of the journal

Frontiers in Public Health

Received: 30 June 2021 Accepted: 14 September 2021

Published: 02 November 2021

Citation:

Qi S, Yin P, Zhang H, Zhang Q, Xiao Y,

Deng Y, Dong Z, Shi Y, Meng J,

Peng D and Wang $Z$ (2021)

Prevalence of Dementia in China in

2015: A Nationwide

Community-Based Study.

Front. Public Health 9:733314.

doi: 10.3389/fpubh.2021.733314

\section{Prevalence of Dementia in China in 2015: A Nationwide Community-Based Study}

\author{
Shige Qi ${ }^{1+}$, Peng Yin ${ }^{1+}$, Han Zhang ${ }^{1}$, Qingjun Zhang ${ }^{2}$, Yize Xiao ${ }^{3}$, Ying Deng ${ }^{4}$, \\ Zhong Dong ${ }^{5}$, Yan Shi ${ }^{6}$, Jun Meng ${ }^{7}$, Dantao Peng ${ }^{8 *}$ and Zhihui Wang ${ }^{1 *}$ \\ ${ }^{1}$ National Center for Chronic and Noncommunicable Disease Control and Prevention, Chinese Center for Disease Control \\ and Prevention, Beijing, China, ${ }^{2}$ Hubei Provincial Center for Disease Control and Prevention, Wuhan, China, ${ }^{3}$ Yunnan Center \\ for Disease Control and Prevention, Kunming, China, ${ }^{4}$ Sichuan Center for Disease Control and Prevention, Chengdu, China, \\ ${ }^{5}$ Beijing Center for Disease Control and Prevention, Beijing, China, ${ }^{6}$ Shanghai Municipal Center for Disease Control and \\ Prevention, Shanghai, China, ${ }^{7}$ Guangxi Center for Disease Control and Prevention, Nanning, China, ${ }^{8}$ China-Japan \\ Friendship Hospital, Beijing, China
}

Objective: This study aims to estimate the prevalence of dementia and Alzheimer's disease $(A D)$ and associated risk factors among the general Chinese population.

Methods: We carried out a nationwide study including 24,117 participants aged 60 years and older in China using a multistage clustered sampling. Dementia and AD were diagnosed according to the fourth edition of the Diagnostic and Statistical Manual of Mental Disorders and the criteria issued by the National Institute of Neurological and Communicative Disorders and Stroke-Alzheimer's Disease and Related Disorders Association. Face-to-face interviews were administered by the trained interviewers to obtain information on demographics, lifestyle factors, and previous diseases.

Results: The overall weighted prevalence of dementia was 4.22\% (95\% Cl 2.27-6.17\%) for people aged 60 years and older, was higher in women than in men and increased with age. Daily tea drinking and daily exercises were the protective factors for both dementia and AD. Engaging in social and intellectual activities was significantly associated with a lower risk of dementia and $A D$.

Conclusions: A large number of population with dementia posed a significant challenge to China where the population is rapidly aging. The increase of public awareness, building more care facilities, and training dementia specialists and professional caregivers are all urgently needed and should be the future priorities of dementia care in China.

Keywords: dementia, prevalence, risk factors, Alzheimer's disease, cross-sectional study

\section{INTRODUCTION}

China is aging much faster than other low- and middle-income countries. WHO estimated that the proportion of the population aged 60 years and over will increase from $12.4 \%$ in 2010 to $28 \%$ in 2040 (1). Accompanying the aging of the society is an increase in age-associated diseases, with dementia playing an important role. The World Alzheimer Report 2015 estimated that there were 
over 9.9 million new cases of dementia each year worldwide, implying one new case every $3.2 \mathrm{~s}$ (2). The Global Burden of Disease Study 2016 reported that the number of patients with dementia in China accounted for $\sim 25 \%$ of the entire population with dementia worldwide (3). A few studies have reported the prevalence of dementia in China, and the estimates remain inconsistent, ranging from 5.0 to $7.7 \%$ for people aged 60 years and older and from 2.0 to $13.0 \%$ for people aged 65 years and older (4). Most of the previous studies were carried out in single cities or a few localities with small sample sizes, and the significant variations might be mainly due to the different diagnostic criteria, instruments used to assess dementia, the time of the study, and a different sampling scheme, and the characteristics of the study participants. A multicity crosssectional study reported that the prevalence was $5.60 \%(3.50-$ $7.60 \%$ ) for people aged 65 years and above in 2013, based on 5,326 study participants (5). A recent meta-analysis summarized 96 studies and reported an overall prevalence of dementia of $5.30 \%$ (4.30-6.30\%) for Chinese population aged 60 years and above (6), which was lower than the estimate for China (6.19\%) and southeast Asia (7.64\%) by the World Alzheimer Report 2015 (2).

In an aging society such as China, an understanding of the current prevalence of dementia and its risk factors is important for policymakers to prioritize patient care and allocate limited health resources. In this article, we reported the prevalence of dementia and Alzheimer's disease (AD) and associated risk factors among the general population aged 60 years and older, based on a large nationwide study carried out in China in 2015.

\section{METHODS}

\section{Study Population}

The Prevention and Intervention on Neurodegenerative Disease for Elderly in China (PINDEC) study was initiated in 2015 aiming to understand the epidemiology of neurodegenerative diseases and associated risk factors among the population aged 60 years and above in China. We used multistage clustered sampling to select the study population. According to the geographic location, population size, and level of economic development, we first selected Beijing, Shanghai, Hubei, Sichuan, Guangxi, and Yunnan as study provinces, representing 20.7\% of the total Chinese population and $22.2 \%$ of the population aged 60 years and above in 2015 (Appendix 1). Within each province, one urban district and one rural county were randomly selected as the study sites (counties or districts). Within each site, one subdistrict in urban areas or one township in rural areas was selected with probability proportional to its size. Within each subdistrict or township, four to eight neighborhood communities or administrative villages were selected with probability proportional to their size. Within each neighborhood community or administrative village, 100200 households with people aged 60 years and above were randomly selected as the study households. In the final stage, study participants were selected based on the inclusion and exclusion criteria. The inclusion criteria are as follows: (1) aged 60 years and above (2) have registered Hukou, and (3) lived in the household for more than 1 year. The Hukou system is a family registration program that serves as a domestic passport in China, regulating population distribution and rural-to-urban migration. All individuals register their Hukou in Public Security Department as a legal document, and the Hukou statistics are normally used as a reliable and stable source to reflect the local demography as it is not biased by a rapidly growing number of the migrant population. Subjects who refused to participate, had a life-threatening illness, were living in hospitals, or were institutionalized were excluded. Figure 1 shows the flow chart of this study. A total of 26,164 people were selected, and 24,117 participated in the survey. The overall response rate was $92.2 \%$. The main reasons for refusal included participants who were too busy, temporarily visited families living in other cities, or they think the survey is not important to themselves.

\section{Data Collection}

Data collection was conducted either in examination centers at local health stations in the residential area of participants or in the household of participants by the trained staff according to a standard protocol, from June to December 2015. A comprehensive questionnaire, including information on demographic characteristics, medical history, and lifestyle factors, was administered by the trained interviewers. Bodyweight, height, and waist circumference were measured according to a standard protocol, and body mass index (BMI) was calculated as the weight in kilograms divided by the height in square meter. Based on the Chinese criteria of the China Obesity Working Group (7), normal weight was defined as $18.5 \leq$ BMI $<24.0$, low weight was defined as BMI $<18.5$, overweight was defined as $24.0 \leq \mathrm{BMI}<28.0$, and obesity was defined as $\mathrm{BMI} \geq 28.0$. Blood pressure was measured at the non-dominant arm three times consecutively with a 1-min interval between the measurements in a seating position after $5 \mathrm{~min}$ of rest using an automated device. Blood samples were collected in all participants after overnight fasting of at least $10 \mathrm{~h}$. Fasting blood glucose (FBG) and serum total cholesterol (TC), low-density lipoprotein-cholesterol (LDL-C), high-density lipoprotein cholesterol (HDL-C), and triglycerides (TG) were measured. Diabetes was defined as (1) a self-reported previous diagnosis by healthcare professionals, and (2) the FBG level of $126 \mathrm{mg} / \mathrm{dl}$ $(7.0 \mathrm{mmol} / \mathrm{L})$ or higher (8). The 2016 Chinese Guideline for the Management of Dyslipidemia in Adults (Chinese guideline) (9) was used to classify the serum TC, LDL-C, HDL-C, and TG levels. High TC was defined as TC $\geq 6.22 \mathrm{mmol} / \mathrm{L}$. High LDL-C was defined as LDL-C $\geq 4.14 \mathrm{mmol} / \mathrm{L}$. Low HDL-C was defined as LDL-C $<1.04 \mathrm{mmol} / \mathrm{L}$ and high TG was defined as TG $\geq$ $2.26 \mathrm{mmol} / \mathrm{L}$. The presence of other diseases was a self-reported previous diagnosis by healthcare professionals.

To ensure the validity and reliability of the data, a stringent quality control protocol was implemented throughout the data collection process. All investigators, interviewers, and laboratory staff underwent intensive training sessions with written and practical exams after the training. Only certified staff were approved to participate in this study and to carry out data collection in the field sites. All questionnaires were administered using a portable PAD with automatic skip pattern and logic checking. All interviews were recorded, and $5 \%$ of the total 


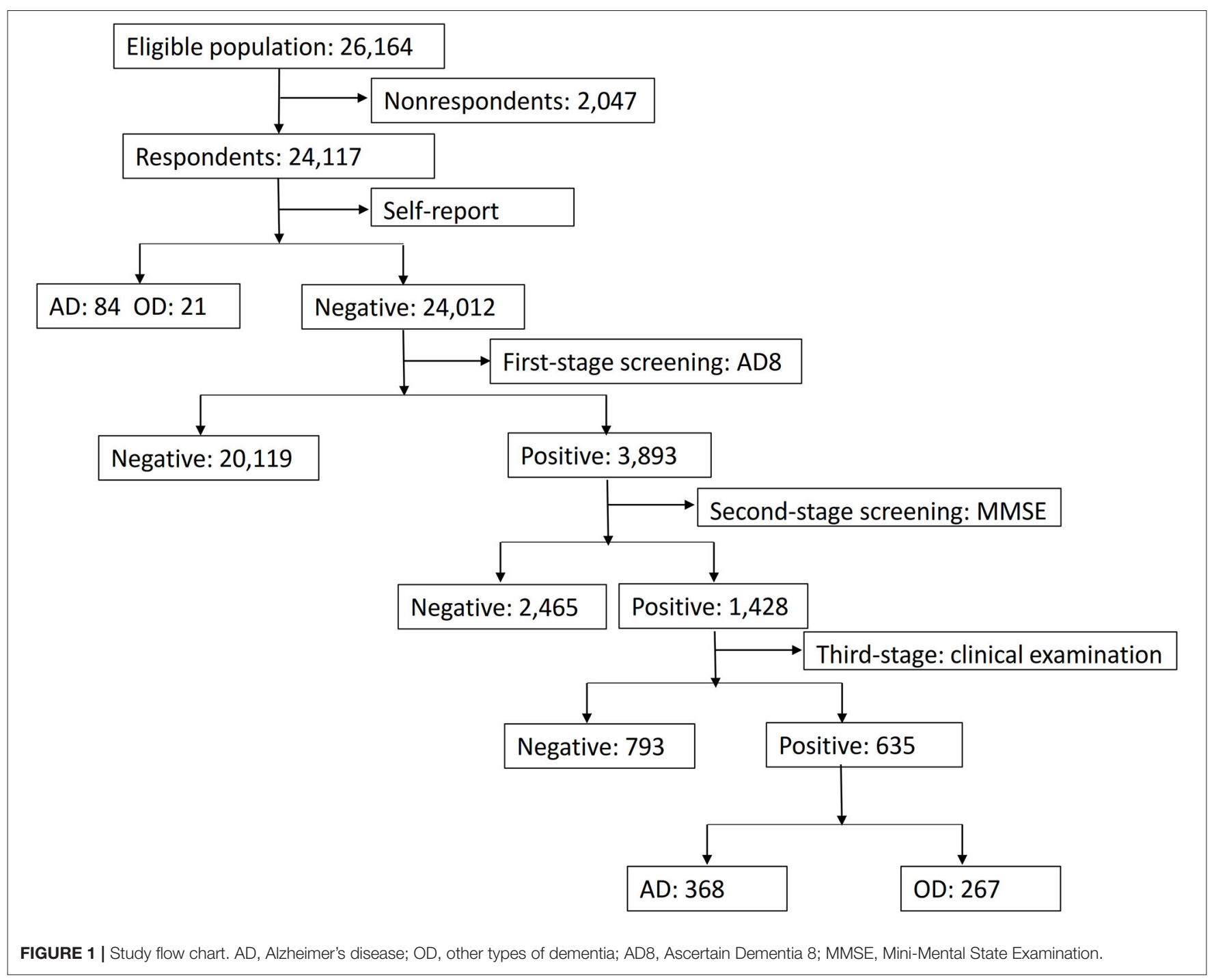

recordings were randomly selected to check consistency and data coding quality.

\section{Dementia and AD Assessment}

When the study participant was selected in the final sampling stage, all participants were asked whether they have been previously diagnosed with dementia by health professionals. Medical records were obtained for those with an affirmative answer, and a self-reported diagnosis was made if the diagnosis was made with clinical examinations in hospitals confirmed by the interviewers. The rest of the participants underwent a three-stage approach for dementia assessment. They were first screened with a Chinese version of the Ascertain Dementia 8 (AD8), which has been proven to be a useful and simple screening tool with good validity in the Chinese population (93.9\% sensitivity and $76.0 \%$ specificity) (10). Participants with an AD8 score of $\geq 2$ were then assessed with Mini-Mental State Examination (MMSE), and cognitive impairment was defined as $\mathrm{MMSE} \leq 17$ for illiterate, $\leq 20$ for primary school, and $\leq 24$ for junior high school and above $(11,12)$. In the final stage, all participants with a cognitive impairment underwent a thorough clinical examination by neurologists. Dementia was diagnosed based on the fourth edition of the Diagnostic and Statistical Manual of Mental Disorders (13). The diagnosis of $\mathrm{AD}$ was made based on the criteria issued by the National Institute of Neurological and Communicative Disorders and Stroke-Alzheimer's Disease and Related Disorders Association (14). A regional expert committee comprised of neurologists at each of the six provinces was available to review all the diagnoses, and a national working committee gathered together to discuss difficult cases until a consensus was reached.

\section{Statistical Analysis}

There were very few missing data in the questionnaires, and they were imputed with median or mean values for the same gender and age group in the dataset. Demographic characteristics of study participants and associated factors were described in means (95\% CIs) for continuous variables 
and percentages (95\% CIs) for categorical variables in the overall population and in the subgroups of sex, location (urban/rural), age, and educational level. The prevalence of dementia and $\mathrm{AD}$ was estimated separately for the overall population and for subgroups. All estimates were weighted to represent the overall Chinese population aged 60 years or older. We calculated sampling weighting, non-response weighting, and post-stratification weighting to derive a final weighing for each study participant. The detailed weighting procedure and the results were shown in Appendix 2. To compare with the prevalence from previous studies, we also estimated the prevalence of dementia for people aged 65 years and over. Multivariable logistic regression was used to examine the association of demographic, lifestyle, and metabolic factors with the odds of dementia and AD. We presented the results for both the crude and fully adjusted models. All $P$-values were twotailed, and the $p$-value of $<0.05$ was considered statistically significant. All statistical analyses were conducted using the SAS system, version 9.4 (SAS Institute, Inc., Cary, NC, USA).

\section{RESULTS}

The characteristics of study participants are presented in Table $\mathbf{1}$. Among the 24,117 people aged 60 years and above included in the analysis, $44.5 \%$ were men, $53.7 \%$ were in urban areas, and $21.1 \%$ were widowed. We found no statistically significant difference between men and women with respect to living in urban/rural areas and living alone or with family. The mean age was 70.5 years (SD 7.0), and the median age was 69.0 years. The current smoking rate was $40.3 \%$ for men and $2.1 \%$ for women. The mean BMI was $23.5 \mathrm{~kg} / \mathrm{m}^{2}$, and $80.1 \%$ had regular exercise. About $70.7 \%$ socialized with neighbors on daily basis, and $92.9 \%$ almost never used the internet. The prevalence of diabetes was 19.0\%, and the prevalence of high TC, high LDL-C, low HDL-C, and high TG was $10.2,7.0,12.1$, and $15.8 \%$, respectively.

The overall weighted prevalence of dementia was $4.22 \%$ (95\%CI $2.27-6.17 \%), 2.04 \%$ (95\%CI $1.54-2.55 \%)$ in men and $6.32 \%$ (95\%CI $2.77-9.86 \%)$ in women, $2.90 \%$ (95\%CI $1.61-$ $4.19 \%$ ) in urban areas, and $5.26 \%$ (95\%CI $2.91-7.60 \%)$ in rural areas. The prevalence increased with age from $1.95 \%(95 \% \mathrm{CI}$ $0.97-2.92 \%)$ in $60-64$ years to $9.46 \%$ (95\%CI $7.11-11.81 \%)$ in the age group 80 years and above. The prevalence of dementia was the highest in the illiterate group, decreased with more years of education but increased again in people with the highest educational level (more than 8 years). The weighted prevalence of $\mathrm{AD}$ was $2.32 \%$ (95\%CI $1.60-3.05 \%$ ), and the pattern in different subgroups was similar to dementia (Table 2). The weighted prevalence of dementia and $\mathrm{AD}$ for people aged 65 years and over was $5.34 \%$, 95\%CI $3.08-7.61 \%$ and $2.94 \%$, 95\%CI $2.10-3.78 \%$, respectively (Appendix 3). There were substantial geographic variations in the weighted prevalence of dementia with the highest in Sichuan province $(6.25 \%, 95 \%$ CI $5.50-7.00 \%)$ and the lowest in Guangxi province (1.05\%, 95\%CI $0.73-1.37 \%$ ) (Appendix 4).

Figure 2 shows the estimated prevalence rate of dementia by different behavioral and metabolic risk factors. The prevalence of dementia was lower in daily tea drinkers than those who did not drink tea on daily basis and was lower in people with daily exercises than those who did not have regular exercise for both men and women. The prevalence of dementia was the highest in low BMI and the lowest in those with the obesity group for women, and the highest in normal BMI, and the lowest in the overweight group for men. The differences were not statistically significant in terms of smoking status and alcohol drinking. We tested the interaction for different risk factors with gender, and the results showed that there were interactions for occupation $(p=0.006)$, BMI $(p=0.011)$, and regular exercises $(p<0.0001)$ with gender, but not for the others.

Table 3 shows the odds ratios (OR) of different risk factors for dementia and AD. Age was a strong risk factor for dementia and $\mathrm{AD}$. The educational level at primary school (6 years) was associated with a lower prevalence rate compared with illiterate people. However, when the educational level is increasing to junior high school or above, the association was no longer significant. Regular tea drinking, daily exercises, daily socializing with neighbors, reading newspapers or books on a daily basis, and daily use of the internet were the protective factors for both dementia and AD. Engaging in intellectual activities was significantly associated with a lower risk of dementia and AD. Smoking was associated with a higher prevalence of AD (adjusted OR 1.51, 95\%CI 1.12-2.04) but not with dementia. Alcohol drinking, the presence of diabetes, and the presence of stroke were associated with a higher prevalence of dementia but not with $\mathrm{AD}$. The presence of chronic obstructive pulmonary disease (COPD) and arthritis was both associated with a higher prevalence of dementia and $\mathrm{AD}$.

Appendix 5 shows the estimated prevalence of dementia using the different forms of dyslipidemia. The prevalence of dementia was higher in men with high TC, high LDL-C, and low HDL-C than those without these diseases. The dementia rate was higher in women with high TG than those without a high TG level. However, when controlling the confounding factors in the logistic regression analysis, the association between dementia and the different forms of dyslipidemia was no longer significant.

\section{DISCUSSION}

In this large-scale community-based survey recently carried out in China, we found that the prevalence of dementia and $\mathrm{AD}$ was $4.22 \%$ (95\%CI $2.27-6.17 \%$ ) and $2.32 \%$ (95\%CI $1.60-3.05 \%)$ in individuals aged 60 years and above in 2015. The prevalence increased with age and was higher in women than in men. Regular tea drinking, daily exercises, and frequent participation in social and intellectual activities were significantly associated with a lower prevalence of dementia and AD.

Systematic review articles all reported the pooled rates of dementia/AD among older adults aged 60 or above, and our estimates $(4.22 \%)$ fall within the range of $3.0-5.3 \%$ reported in these studies $(6,15,16)$. In comparison with the latest study from Jia et al. (4), our estimates on the prevalence of dementia for individuals aged 60 years and above were in the lower range of previous estimates. This might be explained by our study sample. 
TABLE 1 | General characteristics of the study population in 2015, $n$ (\%).

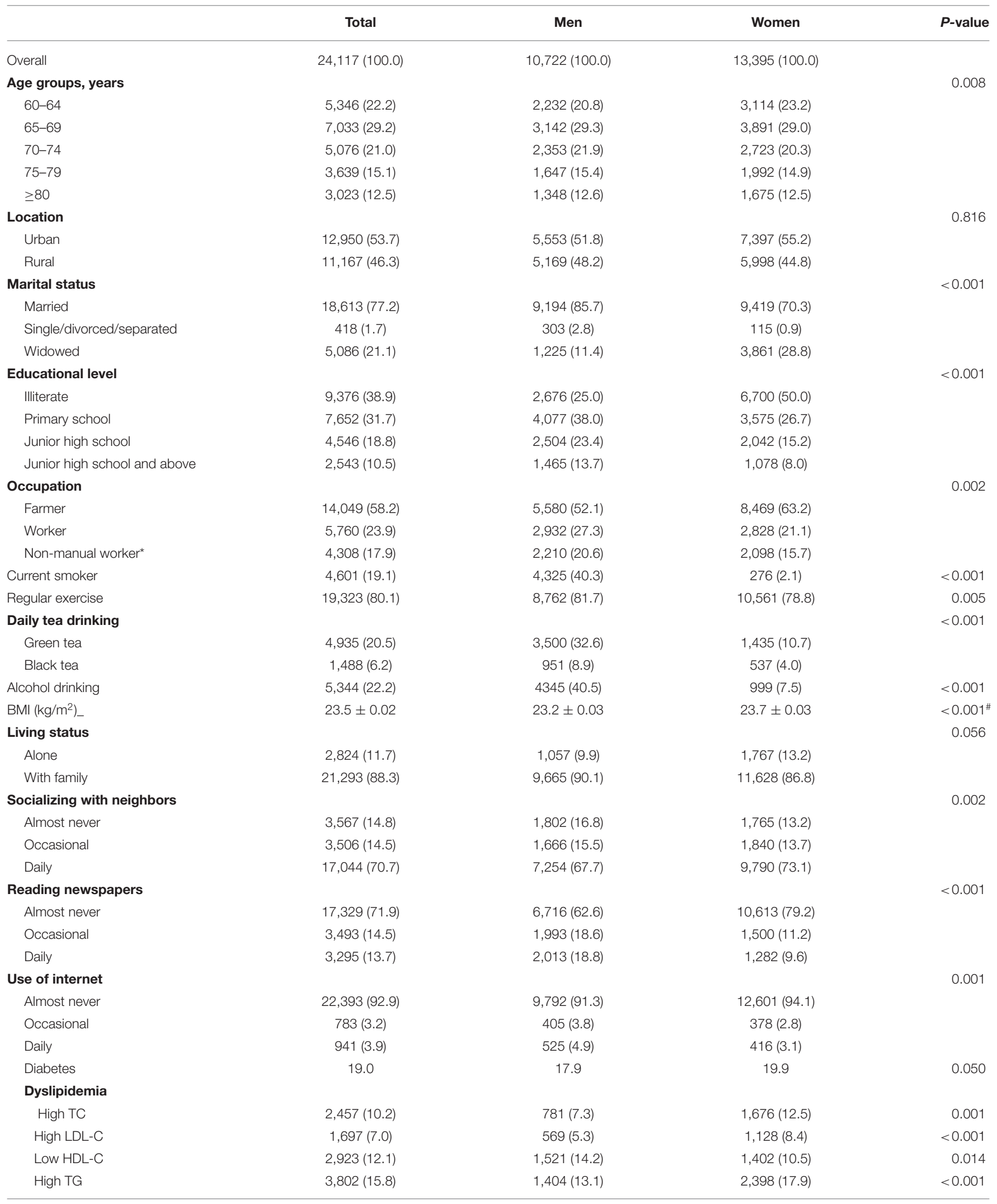

*Non-manual worker includes teacher, researcher, doctors, office workers, and other occupations apart from farmer and worker. \#ANOVA was used to compare the difference of body mass index (BMI) between men and women. 
TABLE 2 | The estimated prevalence of dementia and Alzheimer's disease (AD) in the Chinese population.

\begin{tabular}{|c|c|c|c|c|}
\hline & \multicolumn{2}{|r|}{ Dementia } & \multicolumn{2}{|r|}{ AD } \\
\hline & No. & Weighted prevalence $\%(95 \% \mathrm{Cl})$ & No. & Weighted prevalence $\%(95 \% \mathrm{Cl})$ \\
\hline Overall & 740 & $4.22(2.27-6.17)$ & 452 & $2.32(1.60-3.05)$ \\
\hline \multicolumn{5}{|l|}{ Sex } \\
\hline Men & 220 & $2.04(1.54-2.55)$ & 147 & $1.41(1.03-1.78)$ \\
\hline Women & 520 & $6.32(2.77-9.86)$ & 305 & $3.20(2.00-4.40)$ \\
\hline \multicolumn{5}{|l|}{ Age groups, years } \\
\hline $60-64$ & 73 & $1.95(0.97-2.92)$ & 46 & $1.07(0.71-1.43)$ \\
\hline $65-69$ & 129 & $2.76(1.21-4.31)$ & 65 & $1.14(0.71-1.57)$ \\
\hline $70-74$ & 130 & $3.80(1.88-5.72)$ & 70 & $1.93(1.03-2.84)$ \\
\hline $75-79$ & 188 & $8.31(3.85-12.77)$ & 110 & $3.93(2.78-5.08)$ \\
\hline$\geq 80$ & 220 & $9.46(7.11-11.81)$ & 161 & $6.91(4.60-9.22)$ \\
\hline \multicolumn{5}{|l|}{ Location } \\
\hline Urban & 356 & $2.90(1.61-4.19)$ & 214 & $1.78(0.96-2.59)$ \\
\hline Rural & 384 & $5.26(2.91-7.60)$ & 238 & $2.75(2.04-3.45)$ \\
\hline \multicolumn{5}{|l|}{ Marital status } \\
\hline Married & 485 & $3.36(1.85-4.88)$ & 291 & $1.80(1.27-2.33)$ \\
\hline Single/divorced/separated & 16 & $3.19(2.12-4.26)$ & 12 & $2.54(1.32-3.75)$ \\
\hline Widowed & 239 & 7.37 (4.01-10.73) & 149 & $4.16(2.58-5.74)$ \\
\hline \multicolumn{5}{|l|}{ Education, years } \\
\hline Illiterate & 454 & $7.26(3.02-11.5)$ & 268 & $3.92(2.35-5.50)$ \\
\hline Primary school & 157 & $2.19(1.36-3.03)$ & 102 & $1.22(0.75-1.68)$ \\
\hline Junior high school & 80 & $1.96(1.18-2.75)$ & 47 & $1.02(0.42-1.62)$ \\
\hline Junior high school and above & 49 & $2.32(1.73-2.91)$ & 35 & $1.67(1.17-2.16)$ \\
\hline
\end{tabular}

We randomly selected the study participants from communities across the urban and rural areas in China and represented the general Chinese population. In comparison with other studies, our estimate on Chinese population aged over 60 years was higher than a meta-analysis in 2007 and $2012(15,16)$ and our estimates on Chinese population aged over 65 years $(5.35 \%)$ were in keeping with a five-city study (17) (5.14\%) in 2008-2009 and the multicity study (5) in 2012 (5.6\%). When we further compared the prevalence rate among different 5 -year age groups, we found our estimates for the 65-69, 70-74, and 75-79 age groups were all comparable with the estimates from Jia et al. (17) for both urban and rural areas. However, our estimates for the age group 80 years and above were remarkably lower, resulting in our overall estimates in the lower range of the previous estimates. This can be mainly explained that the elderly participants in our study were relatively healthy because our sample was selected from the general population and excluded those in hospitals or institutionalized.

In consistent with previous studies, we observed a significantly higher prevalence in rural areas than in urban areas, in women than in men, for both dementia and AD. The prevalence of dementia in women was particularly higher than the prevalence of dementia in men, compared with a recent large-scale crosssectional study (18). The magnitude of the differences might be due to different study populations and settings, and further studies are needed to fully address the discrepancy. The prevalence of dementia and $\mathrm{AD}$ among the widowed participants was more than 2-fold of the non-widowed participants, which is also in line with previous reports from both developed countries and China (19-21). Educational level has been identified as an important factor associated with dementia, and illiterate individuals had the highest prevalence rate of dementia and different types of dementia, as indicated in many previous studies $(22,23)$. Jia et al. reported that the prevalence of $\mathrm{AD}$ decreased with more years of education in urban areas in China, while in rural areas, the prevalence was the lowest for those with 7-9 years of education and slightly higher in those with more than 9 years of education (17).

Accumulated evidence from prospective cohort studies proved an increased risk of dementia in smokers. Our study found that smoking was associated with a higher prevalence of $\mathrm{AD}$ but not for dementia. Another multicity study in China found no association between tobacco smoking and the prevalence of $\mathrm{AD}$ and vascular dementia in urban and rural Chinese populations (17). The reason remains unclear, and further studies with biomarker assessment for smoking are needed to illustrate the true association between smoking and dementia in the Chinese population. Previous studies indicated that the association between alcohol consumption and the risk of dementia is dependent on the amount of alcohol $(24,25)$. Although the information on the quantity of alcohol was not obtained in the current study, an analysis on frequency showed that daily alcohol drinking was significantly associated with a higher risk of dementia. For tea-drinking, our result showed 

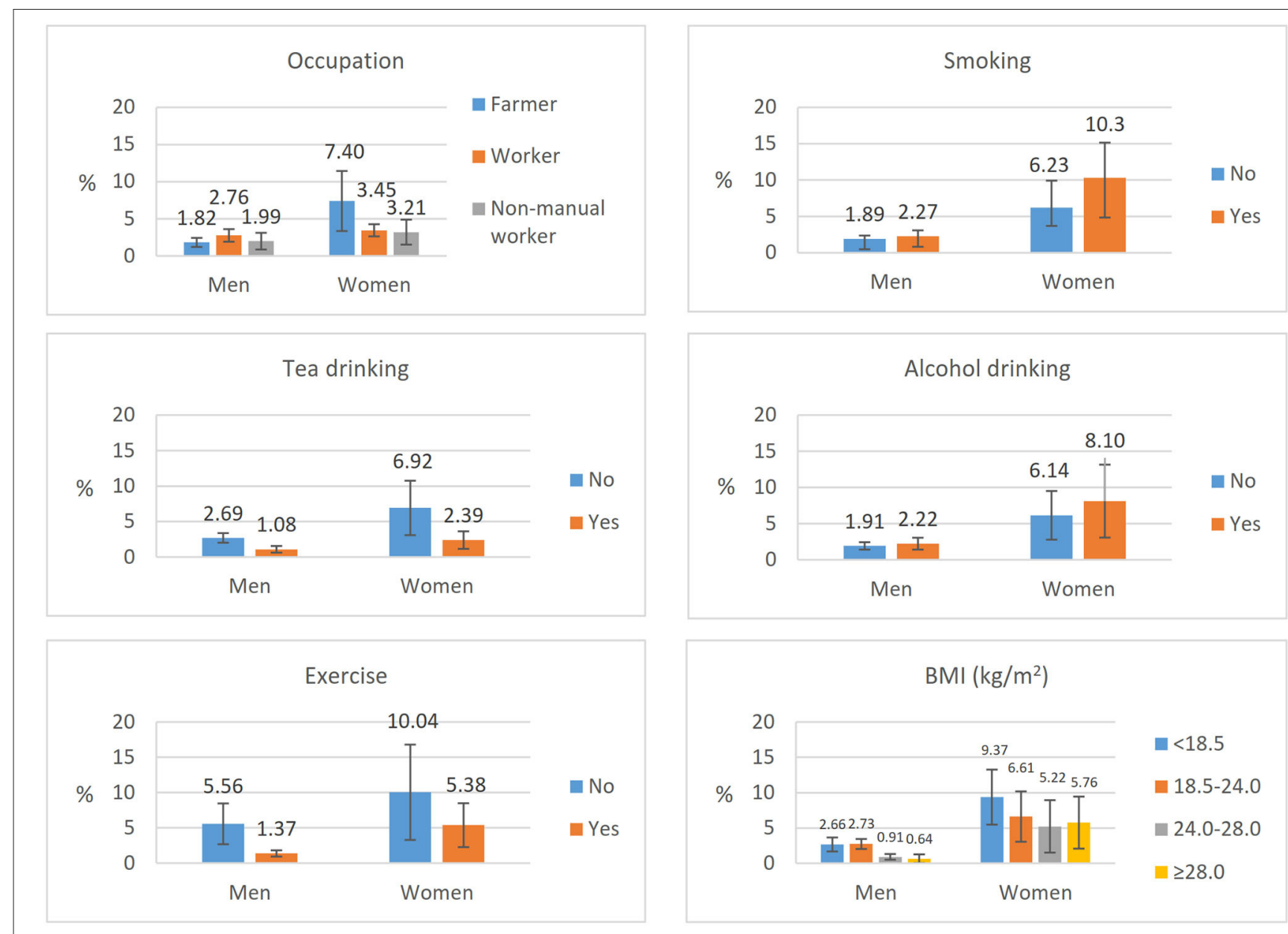

FIGURE 2 | The estimated prevalence of dementia stratified by behavioral and metabolic risk factors.

that both green tea and black tea were the protective factors for dementia and AD. A few studies have reported the hypothesis that green tea intake might reduce the risk for dementia, AD (26). However, whether black tea intake was associated with dementia and $\mathrm{AD}$ needs further research.

Previous studies found that rheumatoid arthritis (RA) increased the risk of dementia, and also reported that a decreased risk of new-onset dementia was seen in patients with RA and was greater among older men, which may be due to the use of the antirheumatic drug $(27,28)$. In our study, the measurement for arthritis only used self-report, and we did not collect the information about arthritis type and medical history. Potential reasons can be further explored in future studies. Consistent with many studies, a higher prevalence of dementia and $\mathrm{AD}$ was seen in patients with COPD (29-31).

The most recent World Alzheimer Report 2019 indicated that $80 \%$ of the general public are concerned about developing dementia at some point, and one in four people think that there is nothing we can do to prevent dementia, and almost $62 \%$ of the healthcare providers worldwide think that dementia is part of normal aging (32). According to a recent national WeChat-based (a popular instant messaging app) survey on public knowledge about dementia in China, the overall correct rate of total dementia knowledge was $63 \%$ and only half of the participants could identify risk factors accurately (33). Their findings indicated that Chinese people have a low level of knowledge about dementia, especially those aged more than 60 years, with low education and living in rural areas, which are exactly the group of the population at the highest risk of dementia. Urgent actions need to be taken to increase public awareness of dementia in China, especially among the vulnerable groups.

Our study confirmed that people with chronic conditions, especially those with diabetes, stroke, COPD, and arthritis, tended to have a higher prevalence of dementia, posing the importance of the prevention and treatment of these chronic diseases. Due to economic difficulties and low awareness of the disease among patients with dementia and their families, $\sim 70$ $80 \%$ of them have not received treatment in China (34). The major challenge associated with the treatment of patients with 
TABLE 3 | Logistic regression analysis of potential risk factors for dementia and AD (odds ratios (OR), 95\%Cl).

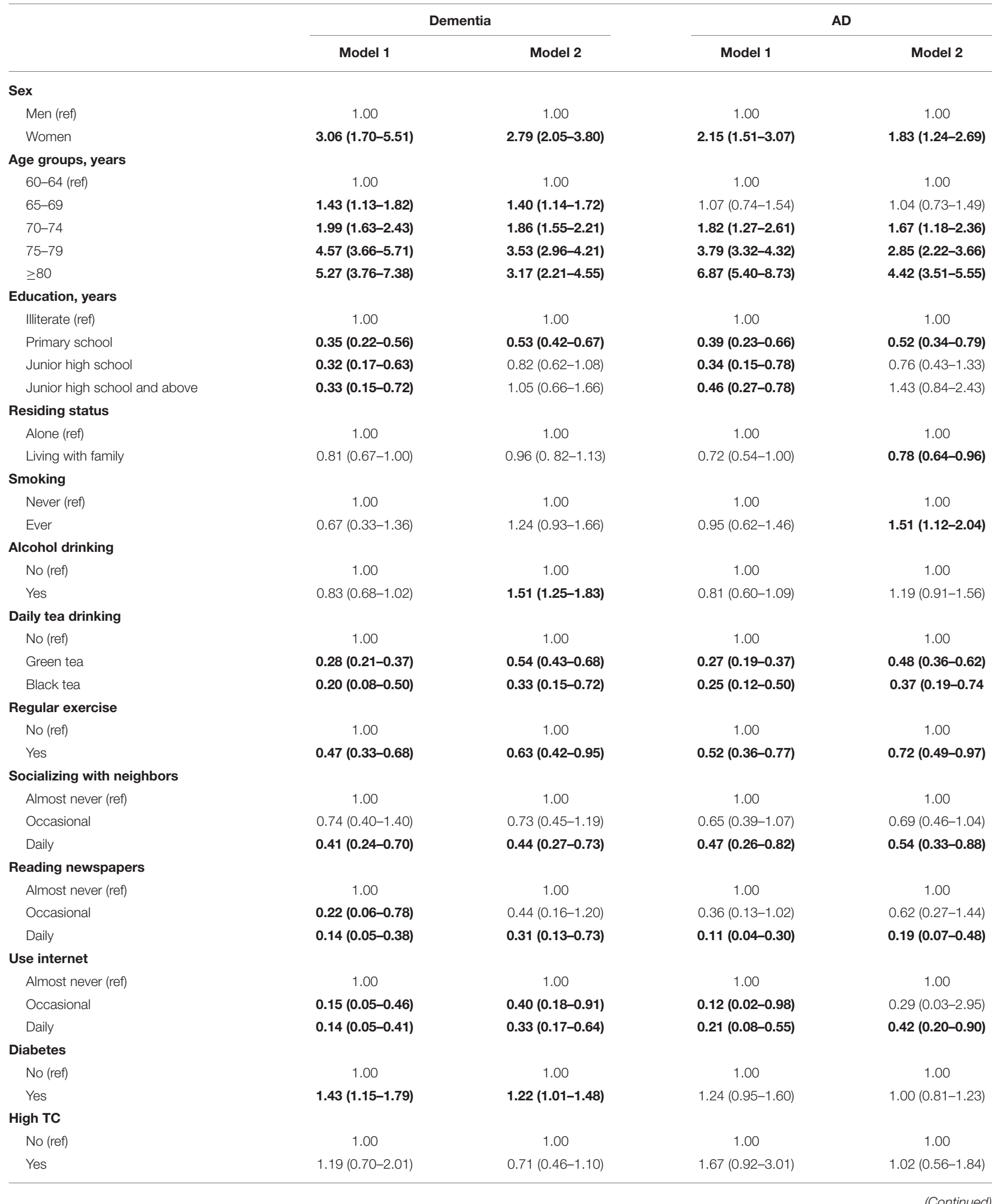

(Continued) 


\begin{tabular}{|c|c|c|c|c|}
\hline & \multicolumn{2}{|c|}{ Dementia } & \multicolumn{2}{|c|}{$A D$} \\
\hline No (ref) & 1.00 & 1.00 & 1.00 & 1.00 \\
\hline Yes & $1.82(1.10-3.00)$ & $1.56(0.98-2.49)$ & $2.39(1.39-4.11)$ & $1.72(0.96-3.06)$ \\
\hline \multicolumn{5}{|c|}{ Low HDL-C } \\
\hline Yes & $1.23(0.77-1.95)$ & $1.17(0.91-1.49)$ & $1.17(0.82-1.66)$ & $0.99(0.74-1.33)$ \\
\hline \multicolumn{5}{|l|}{ High TG } \\
\hline No (ref) & 1.00 & 1.00 & 1.00 & 1.00 \\
\hline Yes & $0.92(0.60-1.40)$ & $0.81(0.54-1.21)$ & $0.95(0.65-1.40)$ & $0.84(0.50-1.41)$ \\
\hline \multicolumn{5}{|l|}{ CHD } \\
\hline Yes & $2.91(1.86-4.57)$ & $2.21(1.62-3.01)$ & $2.23(1.27-3.92)$ & $1.35(0.78-2.32)$ \\
\hline \multicolumn{5}{|l|}{ Cataract } \\
\hline No (ref) & 1.00 & 1.00 & 1.00 & 1.00 \\
\hline Yes & $1.52(0.94-2.43)$ & $1.07(0.75-1.52)$ & $1.86(1.14-3.04)$ & $1.27(0.82-1.98)$ \\
\hline \multicolumn{5}{|l|}{ COPD } \\
\hline No (ref) & 1.00 & 1.00 & 1.00 & 1.00 \\
\hline Yes & $2.06(1.38-3.08)$ & $1.94(1.48-2.54)$ & $2.19(1.54-3.12)$ & $1.84(1.46-2.32)$ \\
\hline \multicolumn{5}{|l|}{ Arthritis } \\
\hline No (ref) & 1.00 & 1.00 & 1.00 & 1.00 \\
\hline Yes & $1.83(1.41-2.36)$ & $1.44(1.23-1.69)$ & $2.01(1.54-2.61)$ & $1.60(1.21-2.11)$ \\
\hline
\end{tabular}

Model 1: Unadjusted crude analysis.

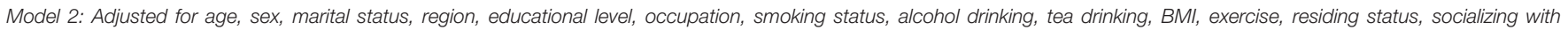
neighbors, reading newspapers, use of the internet, and the presence of some diseases.

Bold values: $P$-value $<0.05$.

dementia in China is the lack of a well-functioned dementia care system, which is further affected by the high cost of care and low levels of public awareness, and poor education among caregivers (35).

We found that daily green tea drinking, regular physical activity, reading books or newspapers, daily use of the internet, and frequent social activities were the protective factors for dementia, which are also reported in studies in China and other countries $(36,37)$. Our findings supported the importance of an active, intellectual, and socially integrated lifestyle among the elderly. In the most recent Healthy China 2030 action plan (38), promoting geriatric health is an important component, and specific actions included encouragement and support of the University for the Elderly, Activity Center for the elderly and Elderly Associations to organize healthy activities across the country.

One important strength of our study is the large sample size and stringent quality control. The study was carried out in 12 counties in 6 provinces across China. A total of 150 interviewers were involved, all of whom attended intensive training sessions and passed exams. A regional expert committee comprised of five provincial-level neurologists confirmed the clinical diagnosis of all cases in each province, and a central expert panel comprised of eight neurologists from the top hospitals in China worked together on difficult cases until a consensus was reached. With strong support from the central and local government of project sites, all the local project activities were well-organized, such as assigning the experienced interviewers to work early morning or night to coordinate with the schedule of participants, offering transportations to the study participants, and the provision of a green channel for all the clinical examinations by local hospitals. All these efforts contributed to the good data quality in this study. The structure of the weighted population was similar to the China Census 2010, proving the representativeness of our study population.

This study has some important limitations. First, temporal associations cannot be inferred due to the cross-sectional nature of the analysis, and recall and response biases might affect the prevalence estimates. Second, despite the weighting use and a high response rate, a limited number of provinces (only six) were selected to represent the national estimates. Older adults from remote rural areas and ethnic minority groups may have been left out. Third, our study participants were selected from the general community residents, with the exclusion of hospital 
and institutional communities, therefore the prevalence might be underestimated due to the institutionalization of some of the elderly individuals. Fourth, we were not able to identify the other subtypes of dementia, such as vascular disease, and cannot depict the whole picture of dementia prevalence. Finally, although we included many potentially associated factors in the logistic regression models, some of the variable categories were still crude. For example, we only asked the frequency of alcohol drinking, and information on the type and units of alcohol was not recorded.

Our study represents the most up-to-date study with large sample size and standard diagnosis in China to estimate the prevalence of dementia and $\mathrm{AD}$ in the general Chinese population. Based on the China National Statistics Bureau, there were 212.42 million Chinese population aged 60 years and over in 2015. If our prevalence estimates are correct, the number of people with dementia would be 8.96 million in China, posing a heavy burden to the family, society, and economy. This finding has serious implications for geriatric health and health services in the rapidly aging society in China. Our study lends further support to increase the awareness of dementia in the general public and to implement effective prevention and control measures on dementia in the Chinese population.

\section{DATA AVAILABILITY STATEMENT}

The raw data supporting the conclusions of this article will be made available by the authors, without undue reservation.

\section{REFERENCES}

1. World Health Organization. China Country Assessment Report on Ageing and Health. (2015). Available online at: https://www.who.int/ageing/publications/ china-country-assessment/en/ (accessed September 28, 2021).

2. Alzheimer's Disease International. World Alzheimer Report 2015-The Global Impact of Dementia: An Analysis of Prevalence, Incidence, Cost and Trends. (2015). Available online at: http://www.alz.co.uk/world-report-2015 (accessed September 28, 2021).

3. GBD 2016 Disease Injury Incidence and Prevalence Collaborators. Global, regional, and national incidence, prevalence, and years lived with disability for 328 diseases and injuries for 195 countries, 1990-2016: a systematic analysis for the Global Burden of Disease Study 2016. Lancet. (2017) 390:1211-59. doi: 10.1016/S0140-6736(17)32154-2

4. Jia L, Quan M, Fu Y, Zhao T, Li Y, Wei C, et al. Dementia in China: epidemiology, clinical management, and research advances. Lancet Neurol. (2020) 19:81-92. doi: 10.1016/S1474-4422(19)30290-X

5. Huang Y, Wang YU, Wang H, Liu Z, Yu X, Yan J, et al. Prevalence of mental disorders in China: a cross-sectional epidemiological study. Lancet Psychiat. (2019) 6:211-24. doi: 10.1016/S2215-0366(18)30511-X

6. Wu Y, Ali G, Guerchet ME, Prina AM, Chan KY, Prince M, et al. Prevalence of dementia in mainland China, Hong Kong and Taiwan: an updated systematic review and meta-analysis. Int J Epidemiol. (2018) 47:709-19. doi: 10.1093/ije/dyy007

7. China Obesity Working Group Data Collection, Analysis Collaborative Group. Predictive values of body mass index and waist circumference to risk factors of related diseases in Chinese adult population. Chin J Epidemiol. (2002) 23:5-10. doi: 10.3760/j.issn:0254-6450.2002.01.003

8. American Diabetes Association. Diagnosis and classification of diabetes mellitus. Diabetes Care. (2010) 33:S62-9. doi: 10.2337/dc10-S062

\section{ETHICS STATEMENT}

The studies involving human participants were reviewed and approved by National Center for Chronic and Noncommunicable Disease Control and Prevention of Chinese Center for Disease Control and Prevention. The patients/participants provided their written informed consent to participate in this study.

\section{AUTHOR CONTRIBUTIONS}

ZW and DP contributed to conception and design of the study. QZ, YX, YD, ZD, YS, and JM contributed to the project execution. $\mathrm{HZ}$ organized the database. SQ performed the statistical analysis and wrote sections of the manuscript. PY wrote the first draft of the manuscript. All authors contributed to the article and approved the submitted version.

\section{FUNDING}

This work was supported by the Major Programs of Public Health of China Ministry of Finance (131091106000150003).

\section{SUPPLEMENTARY MATERIAL}

The Supplementary Material for this article can be found online at: https://www.frontiersin.org/articles/10.3389/fpubh. 2021.733314/full\#supplementary-material
9. Joint Committee Issued Chinese Guideline for the Management of Dyslipidemia in Adults. Chinese guideline for the management of dyslipidemia in adults (revised in 2016). Chin Circul J. (2016) 31:937-50. doi: 10.3969/j.issn.1000-3614.2016.10.001

10. Li T, Wang H, Yang Y, Galvin J, Morris J, Yu X. [The reliability and validity of Chinese version of AD8]. Chin J Intern Med. (2012) 51:777-80. doi: 10.3760/cma.j.issn.0578-1426.2012.10.011

11. Folstein MF, Folstein SE, Mchugh PR. "Mini-mental state": a practical method for grading the cognitive state of patients for the clinician. J Psychiatr Res. (1975) 12:189-98. doi: 10.1016/0022-3956(75)90026-6

12. Zhang M, Katzman R, Salmon D, Jin H, Cai G, Wang Z, et al. The prevalence of dementia and Alzheimer's disease in Shanghai, China: impact of age, gender, and education. Ann Neurol. (1990) 27:428-37. doi: 10.1002/ana.410270412

13. American Psychiatric Association. Diagnostic and Statistical Manual of Mental Disorders.Washington, DC: American Psychiatric Association (1994).

14. Mckhann G, Drachman D, Folstein M, Katzman R, Price D, Stadlan EM. Clinical diagnosis of Alzheimer's disease: report of the NINCDS-ADRDA Work Group* under the auspices of Department of Health and Human Services Task Force on Alzheimer's Disease. Neurology. (1984) 34:939. doi: 10.1212/WNL.34.7.939

15. Dong MJ, Peng B, Lin XT, Zhao J, Zhou YR, Wang RH. The prevalence of dementia in the People's Republic of China: a systematic analysis of 1980-2004 studies. Age Ageing. (2007) 36:619-24. doi: 10.1093/ageing/afm128

16. Zhang Y, Xu Y, Nie H, Lei T, Wu Y, Zhang L, et al. Prevalence of dementia and major dementia subtypes in the Chinese populations: a meta-analysis of dementia prevalence surveys, 1980-2010. J Clin Neurosci. (2012) 19:1333-7. doi: 10.1016/j.jocn.2012.01.029

17. Jia J, Wang F, Wei C, Zhou A, Jia X, Li F, et al. The prevalence of dementia in urban and rural areas of China. Alzheimers Dement. (2014) 10:1-9. doi: 10.1016/j.jalz.2013.01.012 
18. Jia L, Du Y, Chu L, Zhang Z, Qiu Q. Prevalence, risk factors, and management of dementia and mild cognitive impairment in adults aged 60 years or older in China: a cross-sectional study. Lancet Public Health. (2020) 5:e661-71. doi: 10.1016/S2468-2667(20)30185-7

19. Deng J, Cao C, Jiang Y, Peng B, Wang Z. Prevalence and effect factors of dementia among the community elderly in Chongqing, China. Psychogeriatrics. (2017) 18:412-20. doi: 10.1111/psyg.12343

20. Li J, Ogrodnik M, Kolachalama VB, Lin H, Au R. Assessment of the midlife demographic and lifestyle risk factors of dementia using data from the framingham heart study offspring cohort. J Alzheimers Dement. (2018) 63:1119-27. doi: 10.3233/JAD-170917

21. Zhang Z, Zahner GE, Rom An GC, Liu X, Wu C, Hong Z, et al. Sociodemographic variation of dementia subtypes in china: methodology and results of a prevalence study in Beijing, Chengdu, Shanghai, and Xian. Neuroepidemiology. (2006) 27:177-87. doi: 10.1159/000096131

22. Mukadam N, Sommerlad A, Huntley J, Livingston G. Population attributable fractions for risk factors for dementia in low-income and middle-income countries: an analysis using cross-sectional survey data. Lancet Glob Health. (2019) 7:e596-603. doi: 10.1016/S2214-109X(19)30074-9

23. Xu W, Tan L, Wang H, Tan M, Tan L, Li J, et al. Education and risk of dementia: dose-response meta-analysis of prospective cohort studies. Mol Neurobiol. (2016) 53:3113-23. doi: 10.1007/s12035-015-9211-5

24. Sabia S, Fayosse A, Dumurgier J, Dugravot A, Akbaraly T, Britton A, et al. Alcohol consumption and risk of dementia: 23 year follow-up of Whitehall II cohort study. BMJ. (2018) 362:k2927. doi: 10.1136/bmj.k2927

25. Xu W, Wang H, Wan Y, Tan C, Li J, Tan L, et al. Alcohol consumption and dementia risk: a dose-response meta-analysis of prospective studies. Eur J Epidemiol. (2017) 32:31-42. doi: 10.1007/s10654-017-0225-3

26. Ran LS, Liu WH, Fang YY, Xu SB, Wang W. Alcohol, coffee and tea intake and the risk of cognitive deficits: a dose-response meta-analysis. Epidemiol Psych Sci. (2021) 30:e13. doi: 10.1017/S2045796020001183

27. Kronzer VL, Crowson CS, Davis Iii JM, Vassilaki M, Mielke MM, Myasoedova E. Trends in incidence of dementia among patients with rheumatoid arthritis: a population-based cohort study. Semin Arthritis Rheum. (2021) 51:853-7. doi: 10.1016/j.semarthrit.2021.06.003

28. Lin T, Huang J, Pan L, Jong G. Gender-and age-related differences of statin use on incident dementia in patients with rheumatoid arthritis: a Nationwide population-based cohort study. Lipids Health Dis. (2021) 20:1-8. doi: 10.1186/s12944-021-01465-1

29. Xie F, Xie L. COPD and the risk of mild cognitive impairment and dementia: a cohort study based on the Chinese Longitudinal Health Longevity Survey. Dement Geriatr Cogn Dis Extra. (2019) 14:403-8. doi: 10.2147/COPD.S194277

30. Wang Y, Li X, Wei B, Tung T, Tao P, Chien C. Association between chronic obstructive pulmonary disease and dementia: systematic review and metaanalysis of cohort studies. Dement Geriatr Cogn Disord Extra. (2019) 9:250-9. doi: $10.1159 / 000496475$
31. Higbee D, Granell R, Walton E, Korologou-Linden R, Smith GD, Dodd J. Examining the possible causal relationship between lung function, COPD and Alzheimer's disease: a Mendelian randomisation study. BMJ Open Respir Res. (2021) 8:e759. doi: 10.1136/bmjresp-2020-000759

32. Alzheimer's Disease International. World Alzheimer Report 2015-The Global Impact of Dementia: An Analysis of Prevalence, Incidence, Cost and Trends. (2015). Available online at: http://www.alz.co.uk/research/world-report-2019 (accessed September 28, 2021).

33. Public Knowledge About Dementia in China. A national WeChatBased survey. Int J Environ Res Public Health. (2019) 16:4231. doi: 10.3390/ijerph16214231

34. Jia J, Zuo X, Jia XF, Chu C, Wu X. Diagnosis and treatment of dementia in neurology outpatient departments of general hospitals in China. Alzheimers Dement. (2016) 12:446-53. doi: 10.1016/j.jalz.2015.06.1892

35. Zheng C, Xuan Y, Yuetao S, Binbin S, Yi Z, Jiawen L, et al. Challenges of dementia care in china. Geriatrics. (2017) 2:7. doi: 10.3390/geriatrics2010007

36. Marseglia A, Wang HX, Rizzuto D, Fratiglioni L, Xu W. Participating in mental, social, and physical leisure activities and having a rich social network reduce the incidence of Diabetes-Related dementia in a cohort of swedish older adults. Diabetes Care. (2019) 42:232-9. doi: 10.2337/dc18-1428

37. Lee AT, Richards M, Chan WC, Chiu HF, Lee RS, Lam LC. Association of daily intellectual activities with lower risk of incident dementia among older Chinese adults. Jama Psychiat. (2018) 75:697-703. doi: 10.1001 /jamapsychiatry.2018.0657

38. The website of the Government of China. On the Publication of the Healthy China Action 2019-2030. (2019). Available online at: http://www.gov.cn/ xinwen/2019-07/15/content_5409694.htm (accessed September 28, 2021).

Conflict of Interest: The authors declare that the research was conducted in the absence of any commercial or financial relationships that could be construed as a potential conflict of interest.

Publisher's Note: All claims expressed in this article are solely those of the authors and do not necessarily represent those of their affiliated organizations, or those of the publisher, the editors and the reviewers. Any product that may be evaluated in this article, or claim that may be made by its manufacturer, is not guaranteed or endorsed by the publisher.

Copyright (c) 2021 Qi, Yin, Zhang, Zhang, Xiao, Deng, Dong, Shi, Meng, Peng and Wang. This is an open-access article distributed under the terms of the Creative Commons Attribution License (CC BY). The use, distribution or reproduction in other forums is permitted, provided the original author(s) and the copyright owner(s) are credited and that the original publication in this journal is cited, in accordance with accepted academic practice. No use, distribution or reproduction is permitted which does not comply with these terms. 